\title{
Magnetic Field Effect on the Radical Yield of Electron Transfer Reactions Between a Dye Triplet and Heavy Atom Substituted Electron Donors
}

\author{
Ulrich Steiner \\ Institut für Physikalische Chemie der Universität Stuttgart
}

\begin{abstract}
The radical yield in the electron transfer reactions between thionine triplet and various halogenanilines, as measured by kinetic flash spectroscopy in the microsecond time region, decreases by application of magnetic fields up to $4 \mathrm{kGauss}$. The relative magnetic field effect parallels the influence of the heavy atom substituents on the intersystem crossing rate constant in the triplet exciplex supposed to be the primary product of the electron transfer reaction. The magnetic field effect is suggested to be due to differences of the g-factors in the geminate radical pair, originating by dissociation of the triplet exciplex.
\end{abstract}

\section{Introduction}

Reactions involving two paramagnetic species may be influenced by magnetic fields if the reaction possibilities of such pairs depend on their total spin [1]. Magnetic field effects of this kind have been reported for triplet-triplet annihilations [2-4], for reactions of radicals with molecules in excited triplet states [5-7] and for reactions of radicals with radicals $[8,9]$. Magnetic field effects have turned out to be especially useful (see e.g. [10]) for the study of geminate pairs, i.e. pairs originating from one common precursor of definite spin. Such pairs are formed for example in electron transfer reactions of closed shell electron donors (acceptors) with electron acceptors (donors) in an excited singlet or triplet state. Recently several papers appeared, dealing experimentally $[11,12]$ and theoretically $[13-$ 15] with the magnetic field effect on the time dependence of a reaction, in which geminate radical pairs, initially in a total singlet spin state, recombine to yield an excited triplet state. These were mainly concerned with the role of the hyperfine interaction which is also responsible for the CIDNP-effects [10].

The hyperfine coupling provides an efficient mechanism of intersystem crossing in a radical pair, if the four spin states of the pair $\left(S, T_{+}, T_{-}, T_{0}\right)$ are almost degenerate, i. e. if the exchange interaction is sufficiently weak. Applying magnetic fields which cause a Zeeman split of the $T_{+}, T_{-}$levels

Reprint requests to Dr. U. Steiner, Institut für Physikalische Chemie der Universität Stuttgart, Pfaffenwaldring 55, D-7000 Stuttgart 80. larger than the hyperfine coupling, only $S-T_{0}$ mixing remains possible by hyperfine interaction and the effect of a magnetic field in this case is to weaken the hyperfine-induced intersystem crossing. Hence, if we deal with a geminate radical pair, produced in the triplet state and which from energetical reasons can recombine only in the singlet state, the magnetic field should disfavour geminate recombination and correspondingly lead to an increased yield of free radicals.

If the radicals of a pair have different g-factors the magnetic field can influence the spin dynamics of the pair in another way. Since the Larmor frequency of the electron spins is different for the two radicals the magnetic field induces transitions between the $T_{0}$-spin state and the singlet spin state. In this case the magnetic field provides an additional mechanism of intersystem crossing and thus has the opposite effect than when interfering with the hyperfine mechanism. For a radical pair, produced in the triplet state and which can only recombine in the singlet state, the $\Delta g$-mechanism leads to enhanced recombination and hence decreased yield of free radicals. Such an effect was observed by Staerk and Razi Naqvi [16] in a case where the radical pairs were produced by benzophenone triplets abstracting $\mathrm{H}$-atoms from $\mathrm{n}$-hexane.

Deviations of the $g$-factor of electrons bound in atoms or molecules from the free electron value are due to spin-orbit coupling. In aromatic $\pi$-radicals this effect is generally rather small but can be enhanced by heavy atom substituents [17]. The influence of these on the geminate recombination of radical pairs has been observed in CIDNP-experiments [18]. Previously [19] we described a 
heavy atom effect on the radical yield in electron transfer reactions between thionine triplet and monohalogen substituted anilines. The results had to be interpreted by a mechanism whereby a triplet exciplex is formed as the primary electron transfer product. This exciplex decays by the two competing reactions: intersystem crossing to the ground state (sensitive to heavy atom substitution) and dissociation into radicals (insensitive to heavy atom substitution). No magnetic field effect on the intersystem crossing in the exciplex is to be expected [11] since the exchange interaction, causing the triplet-singlet splitting of the exciplex, should be larger than the electronic Zeeman energy in magnetic fields up to some ten kGauss. A magnetic field effect in these systems can arise, however, due to the recombination of geminate radical pairs, originating in the triplet state by dissociation of the triplet exciplex. In this paper we report the flash spectroscopic detection of such magnetic field effects.

\section{Experimental}

Apparatus: Our experimental setup for investigating magnetic field dependent photoreactions is depicted schematically in Figure 1 . The cuvette $C$ (optical pathlength $1 \mathrm{~cm}$ ) containing the sample solution is placed between the poles of a Varian EM 500 eletromagnet (N-S), the field of which can be regulated continuously between 200 Gauss (remanent field) and 4.14 kGauss. To obtain weaker fields and zero field the remanent magnetization is compensated by a current through auxiliary coils wound about the poles of the magnet. The field strength is monitored by means of a hall probe (Bell FTB 1-0415) and a Gaussmeter (Bell, model 615).

The exciting light source is a flash lamp pumped dye laser (electrophotonics, model 43). For the present investigations we used Coumarin 307 (Lamda Physik) as laser dye with a resulting laser wavelength of $500 \mathrm{~nm}$, pulse energy $30 \mathrm{~mJ}$ and pulse width (FWHM) $1.5 \mu$ sec. The laser pulse is attenuated, if necessary, by neutral density filters (GF) and focussed into the cuvette by a lense $\left(L_{3}\right)$ of focal length $f=300 \mathrm{~mm}$. The beam diameter in the cuvette is about $3 \mathrm{~mm}$.

The probe light is provided by a pulsed Xenon lamp (XBO 150, Osram). The probe light beam passes through the excited volume in the cuvette in opposite direction to the laser beam at an angle of $2.5^{\circ}$ against the latter. The probe light beam, the

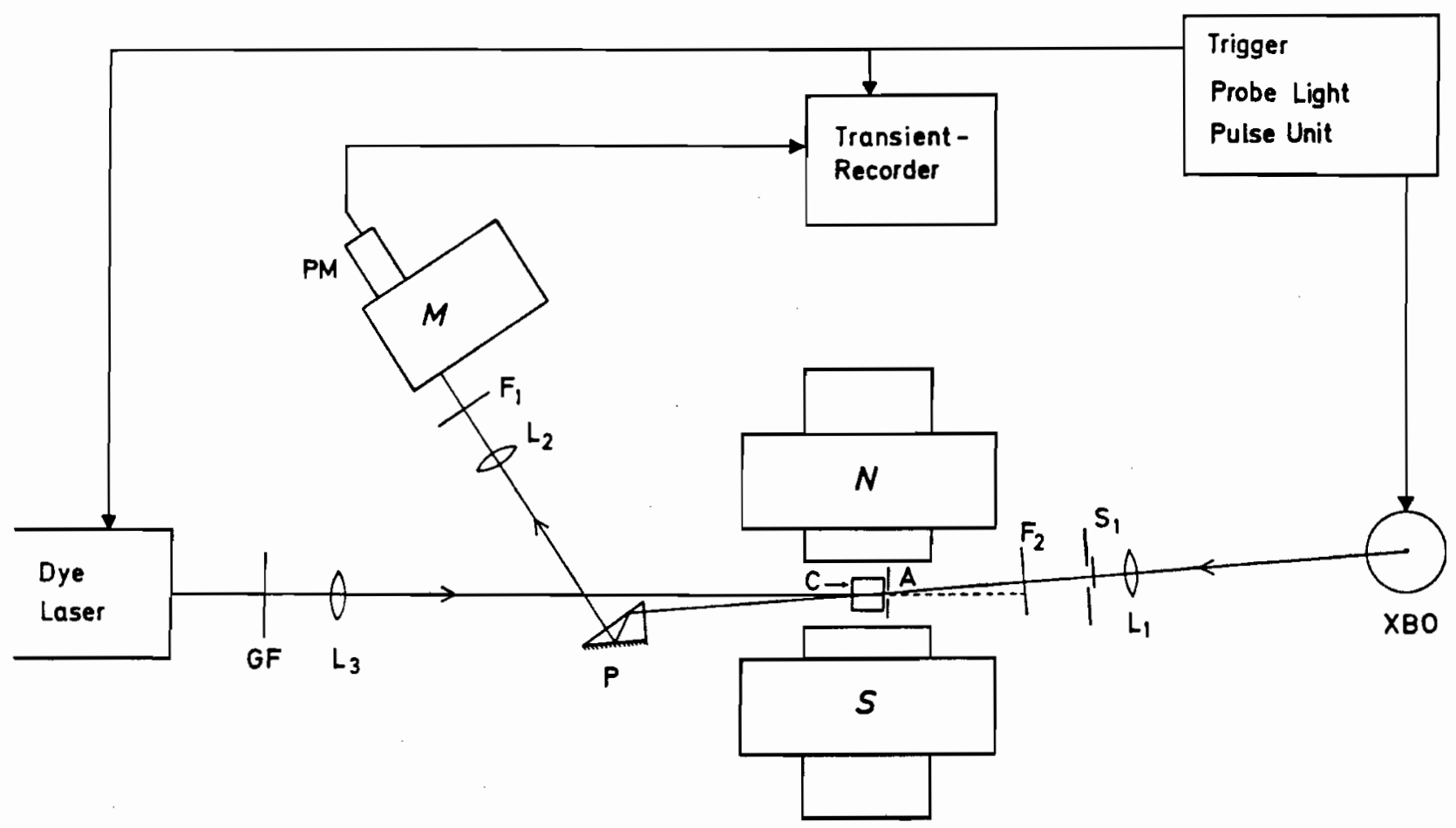

Fig. 1. Experimental setup for observation of magnetic field effects on the formation of photochemical intermediates. A, $1.5 \mathrm{~mm}$ aperture; $C$, cuvette; $F_{1}, F_{2}$, cut-off or interference filters; $G F$, neutral density filter; $L_{1}, L_{2}, L_{3}$, lenses; $M$, monochromator; N-S, electromagnet; $\mathrm{PM}$, photomultiplier; $\mathrm{S}_{1}$, shutter; XBO, pulsed probe light lamp. 
cross section of which is bounded by a $1.5 \mathrm{~mm}$ aperture (A) immediately in front of the cuvette, is deflected to the grating monochromator $M$ (Jarrel Ash, model 82-410) by the prism P. The signal is detected by a photomultiplier PM (Emi 9659, QP) screened against magnetic fields by a $2 \mathrm{~mm}$ sheet of mu-metal. The transient absorption signals were averaged over 12 single excitation pulses, the mean square deviation being $\pm 2 \%$ in such a series.

Materials and methods: Methanol (Merck p. A.) was used as solvent. Thionine was purified according to Endriss [20]. The electron donors used were aniline (Merck p. A., distilled under vacuum), pbromoaniline (Merck p. A.), o- and p-iodoaniline (Merck, Schuchardt $>98 \%$, twice recrystallized from petrolether $\left.\left(40-60^{\circ} \mathrm{C}\right)\right)$ and m-iodoaniline (Merck, Schuchardt $>98 \%$, distilled under vacuum).

The experiments were performed with $3 \times 10^{-5} \mathrm{M}$ (to measure the radical yield) or $1 \times 10^{-5} \mathrm{M}$ (to measure the depopulation of the ground state) solutions of thionine in methanol. The concentration of the electron donors was $2 \times 10^{-3} \mathrm{M}$ so that about $95 \%$ of the dye triplets were depopulated by the bimolecular reaction with the electron donors. The solutions were buffered at a $\mathrm{pH}$ of 8.6 by $0.015 \mathrm{M}$ phenylacetic acid and $0.005 \mathrm{M}$ sodium methylate according to Broser and Fleischhauer [21]. By this measure the free semiquinone radical TH $\cdot$, produced by the electron transfer in the quenching reaction, is rapidly protonated [22] to yield the semiquinone radical $\mathrm{TH}_{2}^{+} \cdot$ which, under the conditions applied, decays to half of its initial concentration in more than 0.5 msec. Furthermore this radical has a strong absorption band in the long wavelength region $\left(\lambda_{\max }=780 \mathrm{~nm}\right)$ where its observation does not interfere with the absorption of the oxidized electron donor radicals. The solutions were deaerated by flushing with nitrogen of oxygen content lower than 5 ppm.

The concentration of radicals produced by a laser pulse of definite energy is proportional to the quantum yield of radical formation only if the absorbing ground state of the dye is weakly depopulated during the exciting pulse [22]. On the other hand the radical concentration should be high to achieve good accuracy of the measurement. As a compromise the laser energy was adjusted by means of neutral density filters so as to yield a radical absorption of about 0.2 corresponding to an about $30 \%$ depopulation of the ground state in a $3 \times$
$10^{-5} \mathrm{M}$ thionine solution. As was shown by numerical calculation, under these conditions there is still good linearity between radical absorption and quan. tum yield of radical formation.

\section{Results and Discussion}

The observation of the magnetic field effect is demonstrated in Figures 2 and 3. Figure 2 shows the transient absorption at the wavelength of maxi-

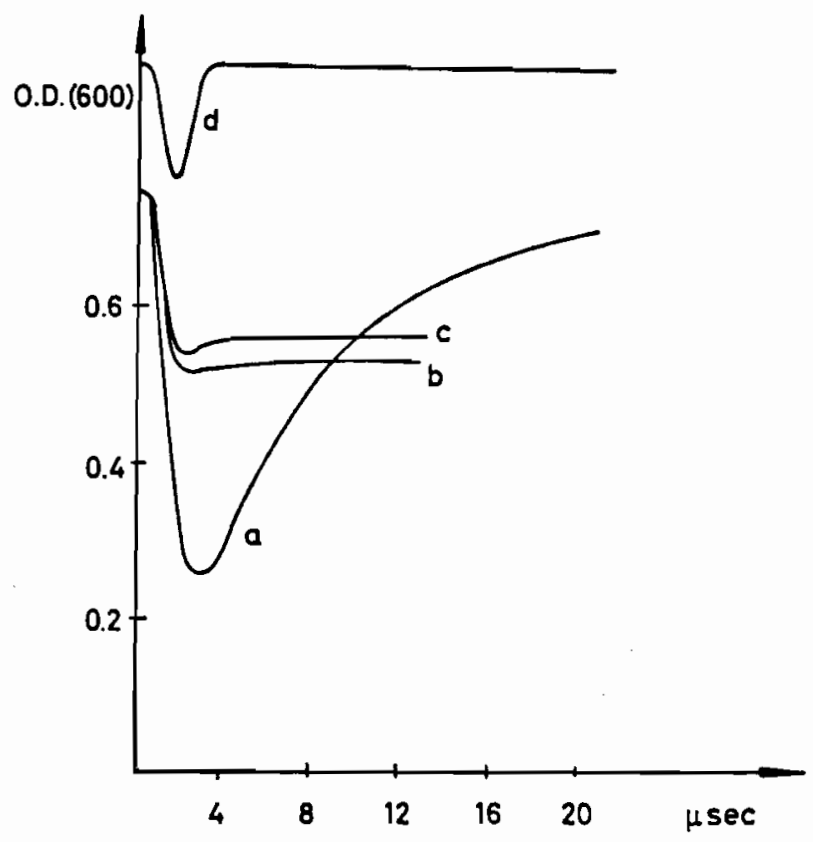

Fig. 2. Magnetic field effect, observed at $600 \mathrm{~nm}$ (maximum of thionine ground state absorption), $\left[\mathrm{TH}^{+}\right]=10^{-5} \mathrm{M}$, solvent methanol, buffered at $\mathrm{pH} 8.6$ (see experimental part). a) Solution without quencher, no influence of mag. netic fields between 0 and $4.14 \mathrm{kGauss,} \mathrm{b)} \mathrm{solution} \mathrm{with}$ addition of $2 \times 10^{-3} \mathrm{M}$ p-iodoaniline, zero magnetic field, c) same solution as b), magnetic field 4.14 kGauss, d) profile of the laser flash, arbitrary units.

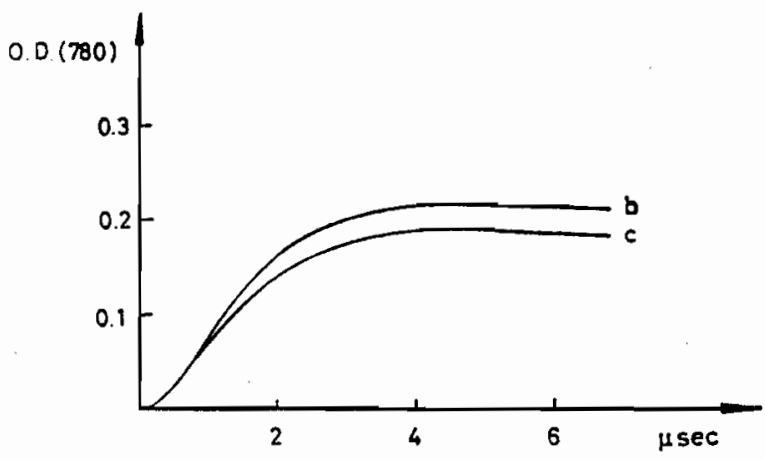

Fig. 3. Magnetic field effect, observed at $780 \mathrm{~nm}$ (naximum of thionine semiquinone $\left(\mathrm{TH}_{2}^{+} \cdot\right)$ absorption, $\left[\mathrm{TH}^{+}\right]=3 \times$ $10^{-5} \mathrm{M}, 2 \times 10^{-3} \mathrm{M}$ p-iodoaniline, solvent methanol, buffered at $\mathrm{pH} 8.6, \mathrm{~b})$ at zero magnetic field, c) with magnetic field of $4.14 \mathrm{KG}$ auss. 
mum absorptivity of the ground state of thionine as a function of time. Trace a is obtained for a solution without electron donor. The primary decrease of optical density corresponds to the depopulation of the ground state due to the population of the triplet state. The secondary increase of the optical density has the same time constant as the decay of the triplet, i. e. it is due to the repopulation of the ground state from the triplet state. Magnetic fields up to $4.14 \mathrm{kGauss}$ have no influence on this signal. Traces $\mathrm{b}$ and $\mathrm{c}$ are obtained under identical conditions as trace a except for addition of $2 \times 10^{-3} \mathrm{M}$ p-iodoaniline. In this case the thionine triplets are quenched within about $0.5 \mu \mathrm{sec}$ in a bimolecular reaction which yields the ground state of thionine and, to a lesser extent, long lived semithionine radicals. Trace $\mathrm{b}$ corresponds to zero field whereas trace $\mathrm{c}$ is obtained with a magnetic field of $4.14 \mathrm{kGauss}$. It can be seen that as an effect of the magnetic field a greater fraction of the triplets is deactivated to the ground state in the quenching reaction.

Figure 3 shows the signals corresponding to trace $\mathrm{b}$ and $\mathrm{c}$ in Fig. 2 (however at a higher dye concentration) at the wavelength of the absorption maximum of the semithionine $\left(\mathrm{TH}_{2}{ }^{+} \cdot\right)$. This diagram shows that the yield of radicals formed in the quenching reaction is decreased by the magnetic field, which complements the effect demonstrated in Figure 2. According to Figs. 2 and 3 the magnetic field effect develops during the excitation flash and therefore can apply only to processes with time constants in the submicrosecond region, i. e. to the primary quenching reaction or to the geminate recombination of radical pairs, but not to a homogeneous. recombination of the radicals.

In Table 1 is shown the relative magnetic field effect $R$ on the radical yield for aniline and various halogenanilines as quenchers of thionine triplet. $R$ is defined by the relation:

$$
R=\left(A_{\mathrm{B}}-A_{0}\right) / A_{0} \times 100
$$

where $A_{\mathrm{B}}$ and $A_{0}$ are the maximum radical absorptions as determined from signals of the kind shown in Fig. 3, with a magnetic field of $4.14 \mathrm{kGauss}$ or at zero field, respectively. In all cases where a magnetic field effect is observed it is negative, i. e. the radical yield is decreased by the magnetic field. This kind of effect suggests that the $\Delta g$-mechanism is operating in the geminate triplet radical pair.

For a quantitative discussion of the results the $g$ -
Table 1. Relative magnetic field effect $R$ a on the radical yield in the reaction of thionine triplet with various electron donors.

\begin{tabular}{lcl}
\hline Donor & $R, \%$ & $n_{\mathrm{isc}} \mathrm{b}$ \\
\hline aniline & $0.0 \pm 2$ & 0.09 \\
p-bromoaniline & $-2.2 \pm 2$ & 1.08 \\
o-iodoaniline & $-3.9 \pm 2$ & 3.76 \\
m-iodoaniline & $-2.9 \pm 2$ & 1.17 \\
p-iodoaniline & $-11.6 \pm 2$ & 8.09 \\
\hline
\end{tabular}

a at a magnetic field of $4.14 \mathrm{kGauss}$,

$\mathrm{b}$ relative intersystem crossing rate constant of corresponding triplet exciplex (from [19]).

factors of the pertinent radicals must be known. At present experimental values are not available, but epr-spectroscopic investigations for their determination are in progress. Qualitatively, however, one can assume that the $g$-factors of the halogenaniline radioals are determined by the strong spin-orbit coupling of bromine and iodine. On the other hand the same influence determines the intersystem cross. ing rate constant of the corresponding triplet exciplexes from which the radical pairs are formed [19]. These rate constants (as relative quantities $n_{\text {isc }}$ ) are also given in Table 1 . Indeed there is a close correlation of $R$ - and $n_{\text {isc }}$-values.

Thus it appears that there is a twofold heavy atom effect (see Fig. 4) : in the triplet exciplex and,

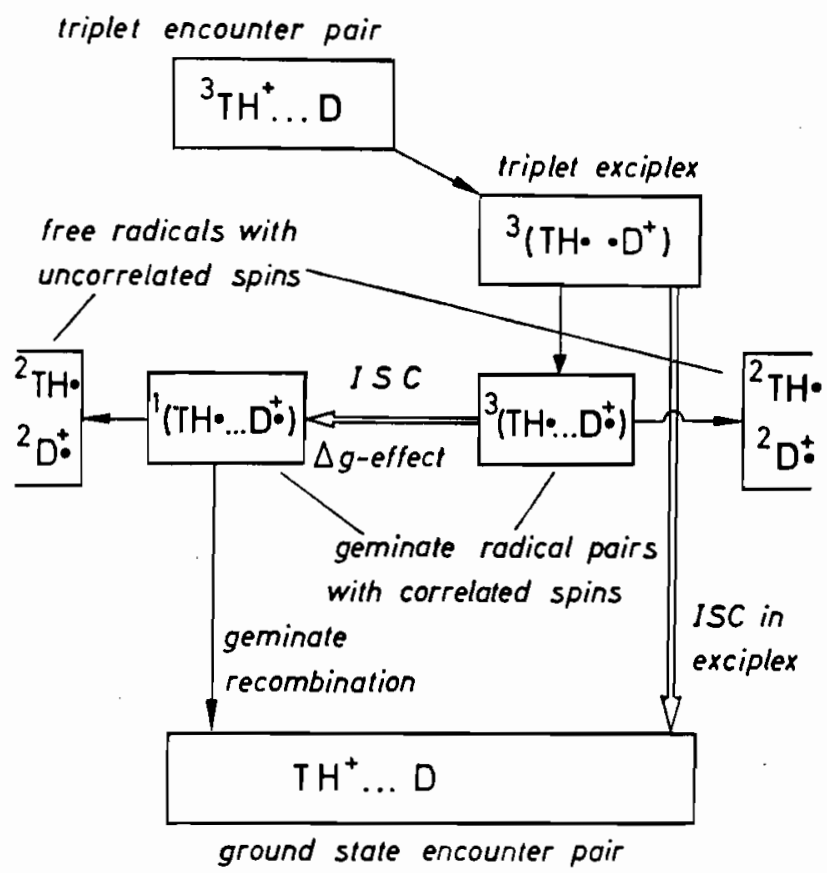

Fig. 4. Reaction scheme explaining the dual heavy atom effect. Processes sensitive to heavy atom substitution are marked by heavy arrows $\left(\mathrm{TH}^{+}=\right.$thionine, $\mathrm{D}=$ electron donor). 
in the presence of a magnetic field, in the geminate radical pair originating by dissociation of the triplet exciplex. We do not expect that the heavy atom substituents induce noticeable intersystem crossing in the geminate radical pair in the absence of a magnetic field. Such a process would give rise to a fast $T_{2}$-relaxation of the radical spins and should cause a corresponding line broadening in the eprspectra. No such extremely broad hyperfine lines have been found, however, in epr-spectra of heavy atom substituted radicals, comparable to the halogenanilines [23].

The reaction scheme (Fig. 4), suggested as an explanation of the observed heavy atom effect and heavy atom induced magnetic field effect, is quite analogous to the reaction scheme suggested by Weller and coworkers [11] for a somewhat different case, where a singlet exciplex is formed primarily in the quenching of pyrene singlet by aromatic amines. Intersystem crossing in this exciplex leads to formation of the pyrene triplet, exciplex dissociation yields a geminate singlet radical pair which undergoes intersystem crossing by the hyperfine interaction, so that geminate recombination, too, can lead to the formation of pyrene triplets. The magnetic field inhibits the hyperfine induced intersystem crossing in the geminate radical pair and thus decreases the yield of geminate triplets.

It is of interest to discuss the role of hyperfine interaction in our systems. The influence of the magnetic field on the hyperfine interaction is probably cancelled by the heavy atom enhanced $\Delta g$-effect. With the unsubstituted aniline as donor radical, however, the hyperfine effect should be dominating. In this case no magnetic field effect is observed (see Table 1) within the accuracy of the experiment. A theoretical treatment [24] by the method of Schulten et al. [14, 25] predicts a magnetic field effect $R \leqq 1.2 \%$ for the system thionine/aniline due to the hyperfine mechanism. This order of magnitude may be obscured within the present precision of our experiments.

For a quantitative theoretical interpretation the magnetic field effect should be determined as a func-

[1] P. W. Atkins and T. P. Lambert, Ann. Rep. Prog. Chem., Ser. A, 72, 67 (1975).

[2] R. E. Merrifield, J. Chem. Phys. 48, 4318 (1968); Acc. Chem. Res. 1, 129 (1968).

[3] R. C. Johnson and E. R. Merrifield, Phys. Rev. B 1, 896 (1970).

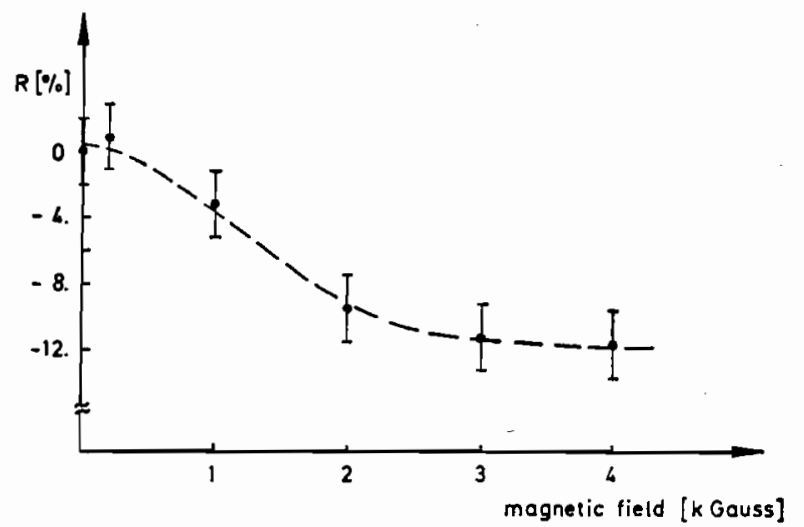

Fig. 5. Dependence of the magnetic field effect $R$ on field strength, for p-iodoaniline as donor (concentrations see Figure 3 ). The dashed line is suggested as a best fit.

tion of the field strength. Within the maximum field strength and accuracy available at present the magnetic field dependence could be quantitatively resolved only for $\mathrm{p}$-iodoaniline which shows the greatest effect. The result, shown in Fig. 5, is somewhat different from other results in the literature, also interpreted by the $\Delta g$-mechanism. Nagakura and coworkers [26] found a square-root dependence of the magnetic field effect on the field strength, in accordance with Kaptein's [27] theoretical treatment. The results of Staerk and Razi Naqui [16], however, indicate a linear magnetic field dependence of the $\Delta g$-effect up to 6 kGauss. Possibly the field dependence observed by us has to be explained by the counteracting effects of hyperfine and $\Delta g$-mechanism. To clarify this point and to establish a correct theory, measurements with increased accuracy and extension to higher fields are in progress, allowing the determination of the quantitative magnetic field dependence of $R$ for all systems in Table 1 .

\section{Acknowledgement}

The author is indebted to Prof. Dr. H. E. A. Kramer for support of this work and critically reading the manuscript. The advice of Dr. D. Miller in translating the manuscript is gratefully acknowledged. Thanks are due to the Deutsche Forschungsgemeinschaft and to Prof. Dr. M. Hauser for placing the dye laser to the author's disposal.

[4] L. R. Faulkner and A. J. Bard, J. Amer. Chem. Soc. 91, 6495 (1969).

[5] ibid. 91, 6496 (1969).

[6] V. Ern and R. E. Merrifield, Phys. Rev. Lett. 21, 609 (1968).

[7] H. Tachikawa and A. J. Bard, Chem. Phys. Lett. 26, 10 (1974) 
[8] B. Brocklehurst, Chem. Phys. Lett. 28, 357 (1974).

[9] R. Z. Sagdeev, K. M. Salikhov, T. V. Leshina, M. A. Kamkha, S. M. Shein, and Y. N. Molin, Exp. Theor. Phys. Letters 16, 422 (1972).

[10] A. R. Lepley and G. L. Closs, Eds., Chemical Induced Magnetic Polarization, John Wiley, New York 1973.

[11] K. Schulten, H. Staerk, A. Weller, H.-J. Werner, and B. Nickel, Z. Physik. Chem. N. F. 101, 371 (1976).

[12] R. Haberkorn and M. E. Michel-Beyerle, Z. Naturforsch. 31 a, 499 (1976) ; M. E. Michel-Beyerle, R. Haberkorn, W. Bube, E. Steffens, H. Schröder, H. J. Neusser, and E. W. Schlag, Chem. Phys. 17, 139 (1976).

[13] Z. Schulten and K. Schulten, J. Chem. Phys. 66, 4616 (1977).

[14] H.-J. Werner, Z. Schulten, and K. Schulten, J. Chem. Phys. 67, 646 (1977).

[15] R. Haberkorn, Chem. Phys. 19, 165 (1977).

[16] H. Staerk and K. Razi Naqvi, Chem. Phys. Lett. 50, 386 (1977).
[17] J. Sinclair and D. Kivelson, J. Amer. Chem. Soc. 90, 5074 (1968).

[18] A. D. Trifunac, Ph. D. Thesis, Chicago 1971; G. L. Closs in [10], p. 106.

[19] U. Steiner and G. Winter, Chem. Phys. Lett. 55, 364 (1978).

[20] W. Endriss, Ph. D. Thesis, University of Stuttgart, 1961.

[21] W. Broser and H. Fleischhauer, Z. Naturforsch. 25 b, 1389 (1970).

[22] U. Steiner, G. Winter, and H. E. A. Kramer, J. Phys. Chem. 81, 1104 (1977).

[23] P. L. Kolker and W. A. Waters, J. Chem. Soc. 1964, 1136.

[24] U. Steiner, to be published.

[25] K. Schulten and P. Wolynes, J. Chem. Phys. 68, 3292 (1978).

[26] Y. Tanimoto, H. Hayashi, S. Nagakura, H. Sakuragi, and K. Tokumaru, Chem. Phys. Lett. 41, 267 (1976).

[27] R. Kaptein, J. Amer. Chem. Soc 94, 6251 (1972). 